\title{
Isolation Enhancement Using a Hybrid Technique in an Eight-Element UWB MIMO Antenna Design
}

\author{
Aicha Mchbal', Naima Amar Touhami', Hanae Elftouh', and Aziz Dkiouak² \\ ${ }^{1}$ Faculty of Sciences, Abdelmalek Essaadi University, Tetouan, Morocco \\ ${ }^{2}$ Natioanl School of Applied Sciences, Abdelmalek Essaadi University, Tetouan, Morocco \\ Corresponding author: M. Aicha (e-mail: aicha.mchbal8@gmail.com).
}

\begin{abstract}
An eight-element multiple input multiple output (MIMO) antenna assembly is designed for Ultra-Wideband (UWB) applications. The antenna configuration is based on a peculiar arrangement of the radiating elements. A defected microstrip structure (DMS) is also introduced on the feedlines. The use of a novel technique, the so-called ports-shift, is here discussed. In the proposed antenna, a protruded ground branch structure is employed in combination of three parasitic stubs so as to enhance isolation and impedance matching over the UWB frequency band, respectively. The results show that the presented antenna exhibits a good impedance matching (Sii) which is about $-10 \mathrm{~dB}$ with a high mutual coupling (Sij) $15 \mathrm{~dB}$, and envelope correlation coefficients (ECC) lower than 0.15 . The antenna also exhibits a good diversity gain (DG) of about $9.5 \mathrm{~dB}$, a good efficiency that varied between $56 \%$ and $91 \%$, and a total active reflection coefficient (TARC) of less than $-20 \mathrm{~dB}$ over the entire UWB frequency band. Which makes it a good candidate for UWB applications.
\end{abstract}

INDEX TERMS Diversity techniques, multiple input multiple output (MIMO), mutual coupling, ultrawideband (UWB).

\section{INTRODUCTION}

Co ontemporary wireless communication systems knows a huge demand of high data rates and reliability simultaneously. To fulfill this demand, UltraWideband (UWB) technology is regarded as a promising solution since the Federal Communications Commission (FCC) approved the commercial use of the bandwidth from $3.1 \mathrm{GHz}$ to $10.6 \mathrm{GHz}$ [1], because of its ability to increase data throughput and improve multi-signals communication reliability thanks to its characteristics which are high data rates, low power level, and low costs [2]. However, the most critical point of this technology is multipath fading [3]. Whereas multiple inputs multiple outputs (MIMO) technique has been introduced as a best solution to enhance UWB technology performance. Therefore, the UWB in association with the MIMO technology could achieve both high data rates and good reliability via multipath fading immunity for current and future needs of wireless communication systems [4].

The MIMO systems concept is based on supporting multiple independent channels at both transmitting and receiving sides over a single antenna configuration. Recently, several works were reported in the literature for UWB MIMO antenna design, however, a considerable amount of these works intend to $2 * 2$ and $4 * 4$ MIMO antenna design. As known from the antenna perspective, in order to get a significant improvement on channel capacity we have to increase the number of transmitter and receiver antennas [5], that is why the demand for increased channel capacity turns the research to higher-order (a greater number of antennas at both transmitter and receiver sides) MIMO antenna design [6]; however, arranging multiple antenna elements in a confined space will lead to poor isolation between adjacent radiators [7]. As known in literature, in order to obtain a good isolation between elements of an antenna assembly, the distance between those elements should be higher than a half-wavelength $(d>\lambda / 2)$ [8]. So, as mentioned in order to get a good isolation we can simply have a high distance between radiators. However, this leads to obtain a bulky system which is not desired by wireless communication systems. That is why while designing a MIMO antenna array, we should take into consideration two parameters, mutual coupling between radiators and antenna size. To overcome this issue, we can use two solution categories:

The first category is to implement decoupling structures such as: parasitic elements, metamaterials, defected ground structures, decoupling networks, electromagnetic band gap, neutralization lines, and slit pattern [9], etc.; however, these latter are characterized by their relatively large size which will lead to obtain a bulky MIMO antenna system, which is not desired. The second one is to apply diversity techniques 
which include spatial, polarization, and pattern diversity. Note that mutual coupling suppressing based on pattern diversity is very limited for multi-antenna applications [10]. In this work, a novel eight-port MIMO antenna assembly for UWB application is investigated. The novelty in the proposed MIMO antenna design is the combination between several decoupling techniques on one design, this is why it is called a hybrid technique. The antenna design based on spatial diversity as well as orthogonal and cross polarization employment to ensure an acceptable port-toport isolation. These techniques are combined with a shiftport technique which is in its turn based on the substrate extension, as well as a protruded ground branch implementation that intend to suppress the electromagnetic coupling among orthogonally polarized and side-by-side arranged radiating elements, respectively. In addition, a DMS (Defected Microstrip Structure) in association with one horizontal and two vertical stubs are loaded in the feedlines as well as on the bottom face of the proposed antenna array to cover the entire UWB frequency band with an improved impedance matching. The organization of this paper is arranged as follow: in the first paragraph, a complete discussion of the proposed antenna design process is presented. Paragraph 2 presents the fabrication process and measured results. Paragraph 3 evaluate the antenna diversity performance, paragraph 4 compares the proposed MIMO antenna performance with reported ones, and paragraph 5 concludes the manuscript.

\section{DESIGN PROCESS DISCUSSION}

\section{A. SINGLE ANTENNA DEIGN}

A full description of the adopted single-element owl-shaped antenna design process is discussed in this section. The adopted antenna is mounted on the top face of a low cost $26 * 15.5 * 0.8 \mathrm{~mm}^{3} \quad$ FR-4 substrate with a relative permittivity 4.3 and a loss tangent of 0.025 . Note that the adopted antenna has evolved from an owl-shaped $2 * 2$ MIMO antenna in [11], and it is simulated in the wellknown 3-D electromagnetic simulator CST MWS (Microwave Studio). Table I illustrates the optimized dimensions of the proposed owl-shaped single antenna element.

Basically, the antenna element has evolved from a simple rectangular patch antenna. With the aim of covering a wide bandwidth, a partial ground plane with different defects has been employed. An arc-shaped structure as well as a semicircle with an open-ended slot are introduced on the patch, in order to extend the operating frequency band and to shift the lower frequency. Furthermore, a defecting has been done on the ground plane so as to enhance the impedance matching and isolation simultaneously. Fig.1 depicts the adopted single element antenna.

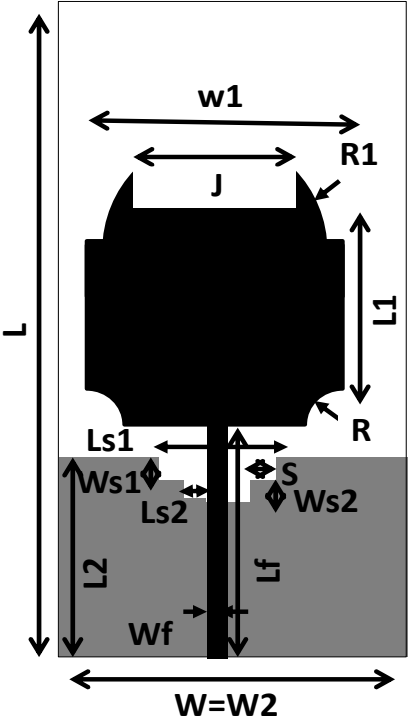

FIGURE. 1 The adopted single patch antenna

TABLE I. The optimized dimensions of the adopted single antenna in ( $\mathrm{mm}$ )

Parameter Value Parameter Value

\begin{tabular}{cccc}
\hline $\mathrm{L}$ & 31 & $\mathrm{~W}$ & 15 \\
$\mathrm{~L}_{1}$ & 8.3 & $\mathrm{~W}_{1}$ & 10 \\
$\mathrm{~L}_{2}$ & 8 & $\mathrm{~W}_{2}$ & 11 \\
$\mathrm{~L}_{\mathrm{f}}$ & 9 & $\mathrm{~W}_{\mathrm{f}}$ & 1 \\
$\mathrm{Ls} 1$ & 4 & $\mathrm{Ws} 1$ & 0.8 \\
$\mathrm{Ls} 2$ & 1 & $\mathrm{Ws} 2$ & 1 \\
$\mathrm{~J}$ & 6 & $\mathrm{~S}$ & 0.5 \\
$\mathrm{R}$ & 2.5 & $\mathrm{R} 1$ & 5 \\
\hline
\end{tabular}

\section{B. 2*2 MIMO ANTENNA ASSEMBLY}

A profound study of a $2 * 2$ MIMO antenna three configurations is accomplished in this section. This investigation is based on three mutual coupling comparison of three different designs taking into accounts the antenna elements arrangement. Note that the first design is composed of two face-to-face placed radiators, the second configuration consists of using two orthogonally placed antenna elements, while the third configuration is composed of two side-by-side placed MIMO antenna elements which is based by its turn on spatial diversity technique. Fig.2(a), (b), and (c) illustrates the 2*2 MIMO antenna three configurations. 

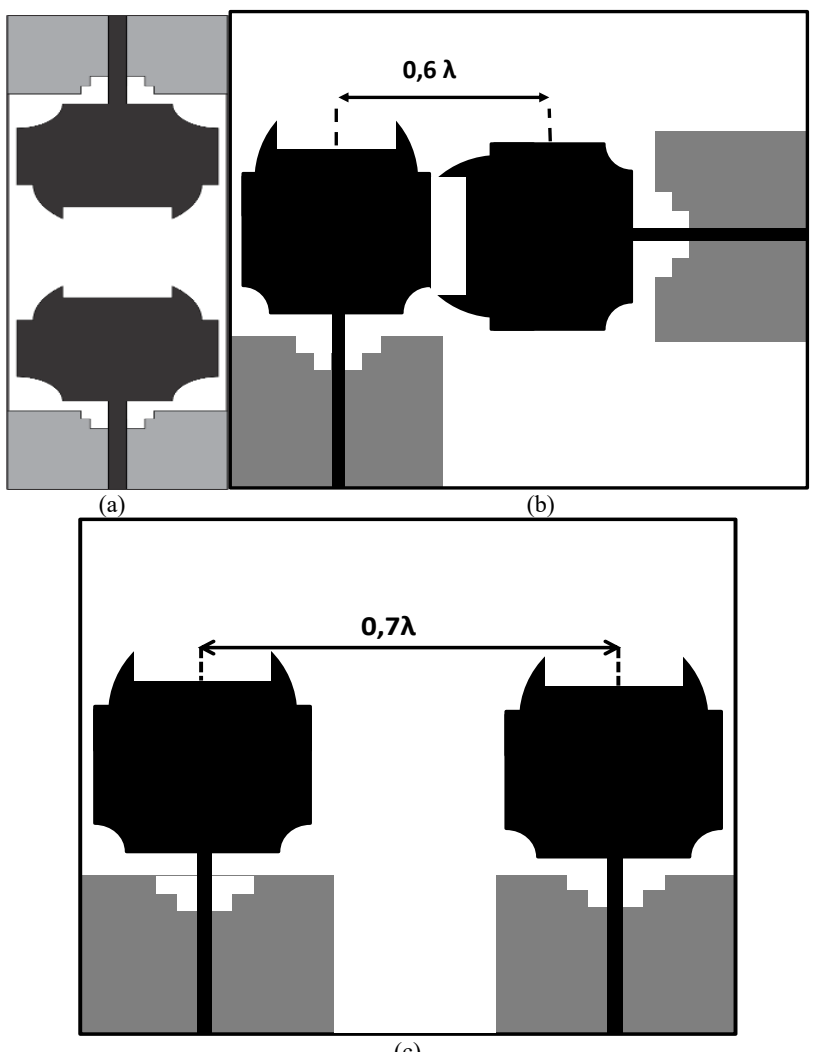

FIGURE. 2 The proposed $2{ }^{\star} 2$ MIMO antenna designs, (a) first design, (b)

second design, (c) third design

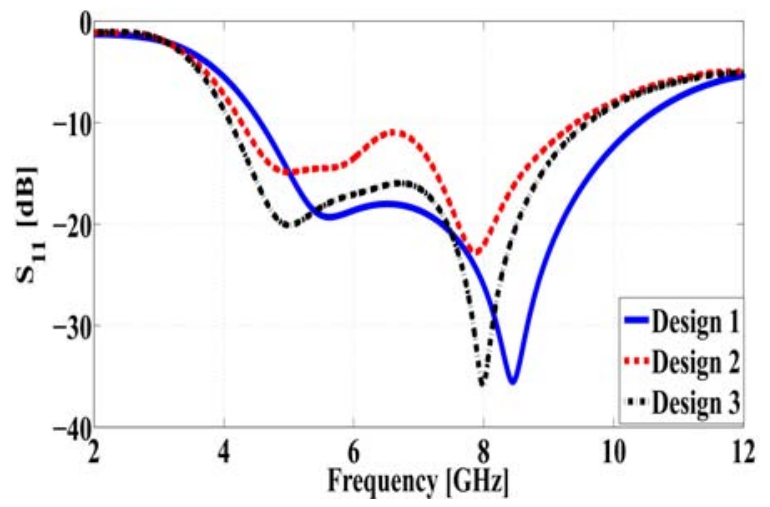

(a)

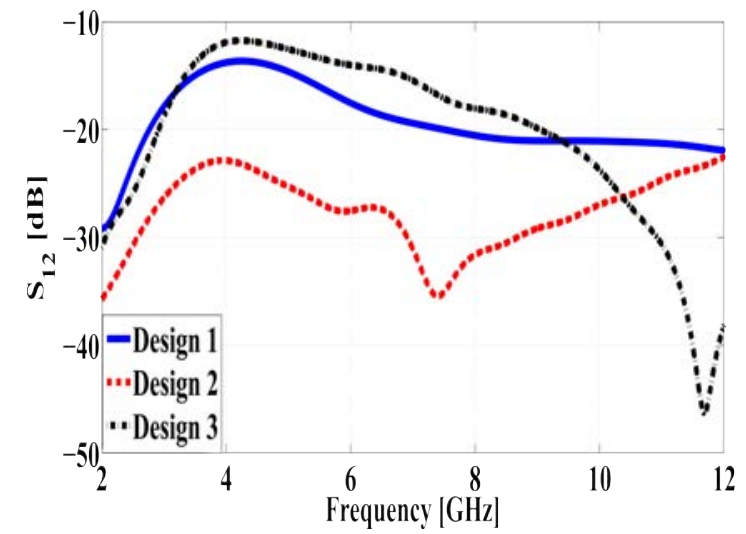

(b)

FIGURE. 3 Simulated S-parameters of the proposed three configurations, (a)

reflection coefficients, (b) mutual coupling

As depicted in Fig.3 (a) and (b) that represents the comparison of the simulated impedance matching and the electromagnetic coupling of the three configurations, simulated results show that configuration 2 that belongs to orthogonal polarization, exhibit a low mutual coupling compared with configuration 1 and 3 , which prove the configuration 2 efficiency and utility at its incorporating level on high order MIMO antenna system.

\section{C. $8 * 8$ MIMO ANTENNA ARRAY}

Based on the predone study of $2 * 2$ MIMO antenna array configurations that proves the orthogonal polarization diversity technique efficiency compared to spatial and faceto-face polarized diversity. The orthogonal polarization technique was selected to be employed on the proposed eight-element antenna design, so as to provide an acceptable port-to-port isolation. The design first step is a study of the used printed circuit board (PCB) dimensions, taking into consideration that the chosen dimensions could support eight-element UWB MIMO antenna. Therefore, a FR-4 substrate with $68 \mathrm{~mm} \times 40 \mathrm{~mm} \times 1.6 \mathrm{~mm}$, a 4.3 relative permittivity, and a 0.027 of loss tangent is chosen.

The proposed eight-ports MIMO antenna consists of fourpair monopole antennas which are placed in a symmetrical way at the four middle edges of the main board. So, based on simulation results of the proposed MIMO antenna first design a narrow frequency band has been covered which does not response to the aim of this work which is to design an eight-element MIMO antenna that intend to UWB applications. Furthermore, despite of the edge-to-edge small separation, an acceptable inter-elements isolation is realized, except between ports ( 1 and 2$)$, ports (1 and 6), and ports (5 and 6$)$ that show a low isolation.

As a solution to this, a DMS is etched on the feedlines in order to provide an enhanced impedance matching and extend the operating frequency band; because as known, the electromagnetic field for a microstrip line is concentrated around the conductor strip. So, the effect of a DMS can be generalized on the modification of current distribution at the microstrip level by increasing the electric length of a microstrip line, which leads by its turn to resonance creation at certain frequency of interest.

Fig.4 illustrates the effect of the etched DMS on the impedance matching. As shown in this figure, a high improvement at the adaptation level has been realized, since before the DMS employment the antenna was not adapted over approximately all the frequency band, while when the DMS is etched on the feedlines, a remarkable enhancement has been achieved in which the frequency band extends from 3.66-5.58 to $3.22-10.31 \mathrm{GHz}$ with a reflection coefficient of about $<-10 \mathrm{~dB}$. 
Further, in order to ensure a low inter-element mutual coupling between orthogonally polarized radiators, substrate extension at the horizontal axis level which is based on ports shift is done. It is obvious from Fig.5(a) and (b) which depicts the simulated mutual coupling before and after the used ports shift technique, that this approach achieves about $20 \mathrm{~dB}$ isolation between orthogonal ports (see S16) across the whole band, while to suppress the electromagnetic coupling between the side-by-side placed antenna elements by separating the current flowing through the common ground planes, a protruded ground branch is introduced between the parallel radiating elements on the bottom face of the presented MIMO antenna system which helped to provide a good isolation by suppressing undesired induced currents between parallel arranged radiating elements in addition to improve and extend the impedance matching. Finally, one narrow horizontal stub and two narrow vertical ones are loaded on the surface of the ground plane so as to enhance the impedance matching over the lower frequency band. Fig.6 (a) and (b) shows the simulated s-parameters after the utilization of the aforementioned mechanisms, as shown in this figure, the lower frequency band is shifted from more than $3.22 \mathrm{GHz}$ to $2.93 \mathrm{GHz}$ to finally cover the UWB frequency band which ranges from 3.1 to $10.6 \mathrm{GHz}$ with an isolation of about $15 \mathrm{~dB}$.

Fig.7 represents the proposed UWB MIMO antenna array final design which is characterized by an overall dimension of $68 \mathrm{~mm} \times 100 \mathrm{~mm} \times 1.6 \mathrm{~mm}$ and a unit cell antenna element size of about $22 \mathrm{~mm} \times 11 \mathrm{~mm}$. Table II illustrates the optimal dimensions of the proposed UWB MIMO antenna.

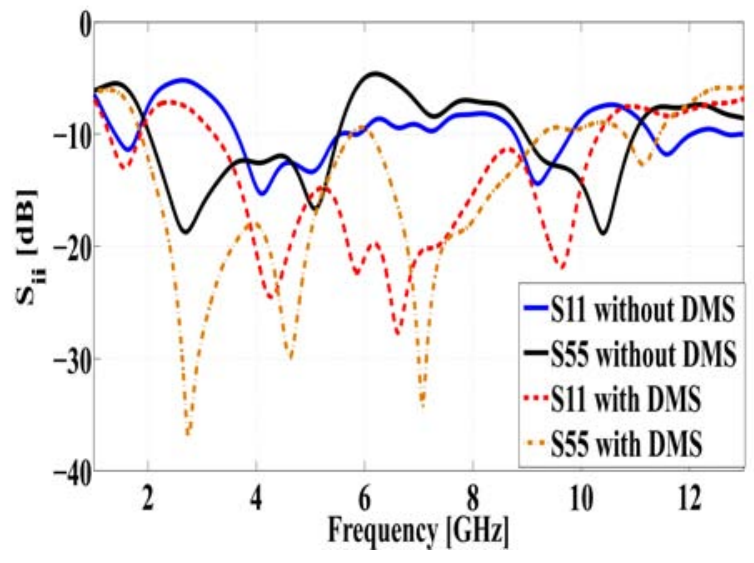

FIGURE.4 Reflection coefficients comparison with and without the defected microstrip structure (DMS)

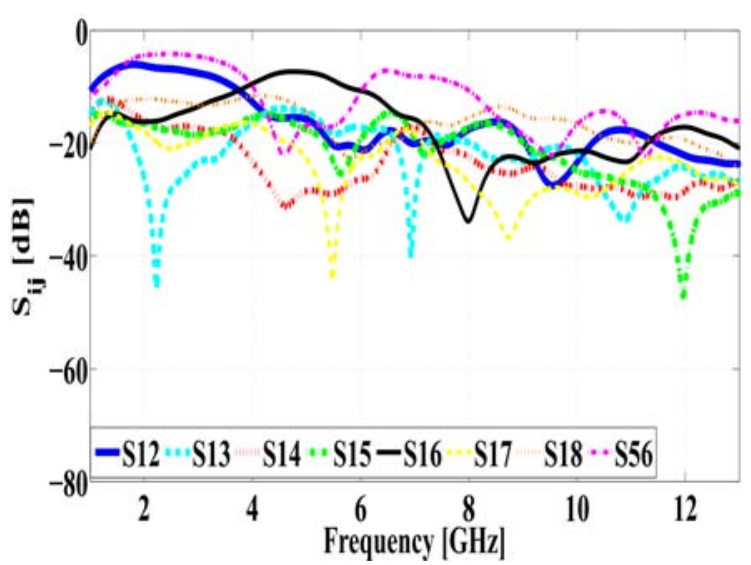

(a)

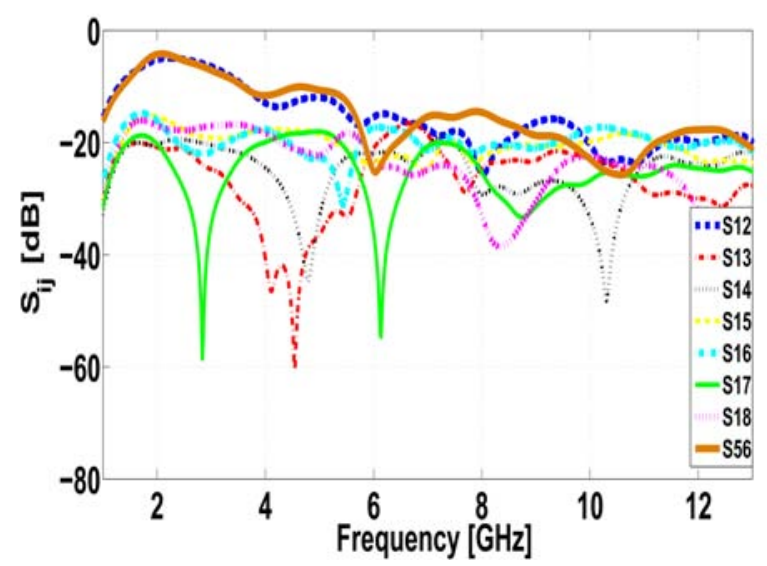

(b)

FIGURE.5 Simulated mutual coupling comparison, (a) before ports shift utilization, (b) after ports shift utilization

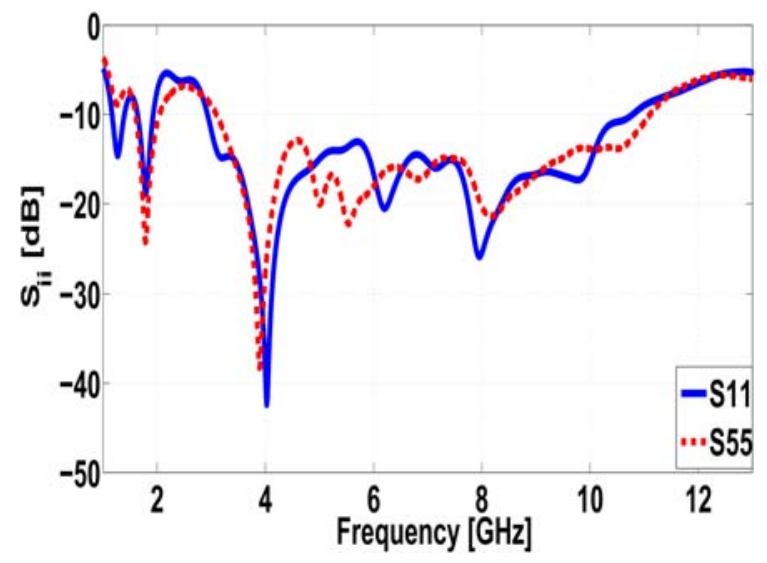

(a) 


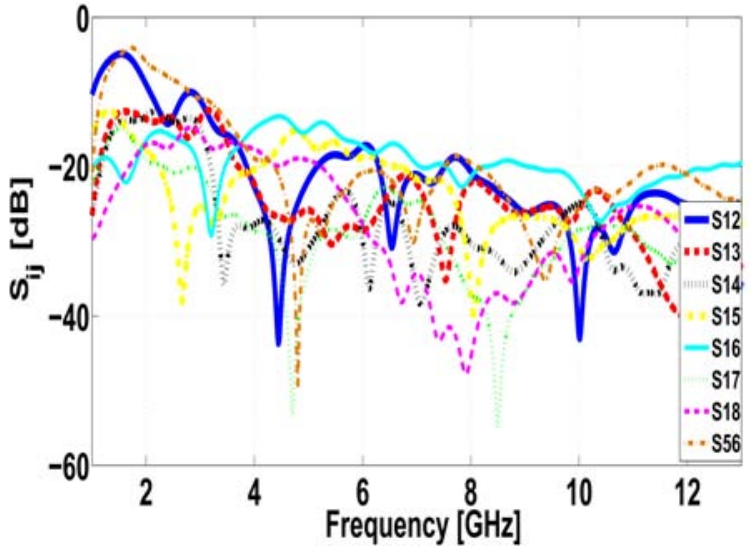

(b)

FIGURE. 6 Simulated s-parameters with the horizontal and vertical stubs, (a) impedance matching, (b) electromagnetic coupling

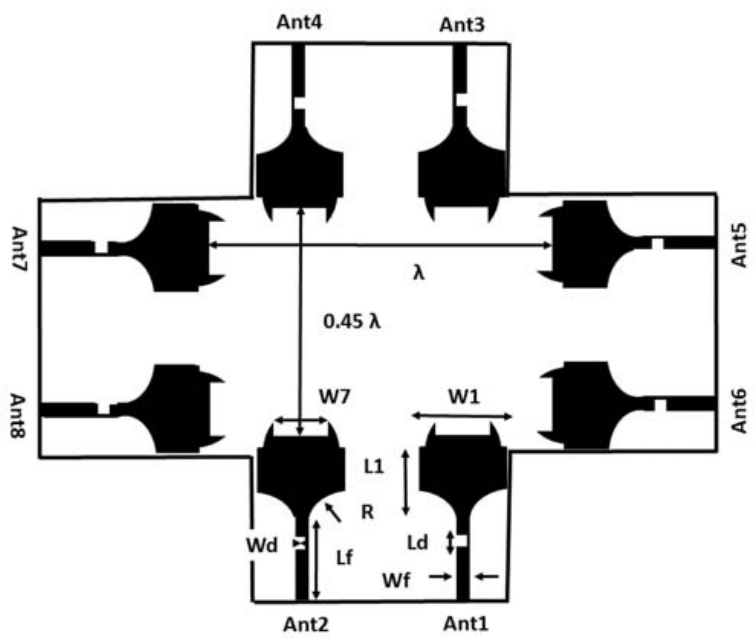

(a)

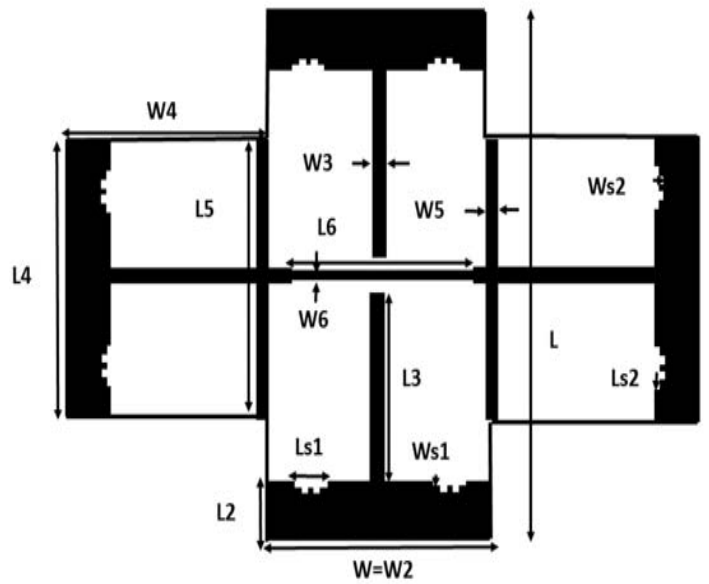

(b)

FIGURE. 7 The presented UWB eight-element MIMO antenna final design, (a) top face, (b) bottom face
TABLE II. The Optimized Dimensions of the Studied UWB MIMO Antenna in $(\mathrm{mm})$

\begin{tabular}{cccc}
\hline Parameter & Value & Parameter & Value \\
\hline L & 68 & W & 40 \\
L1 & 9 & W1 & 11 \\
L2 & 8 & W2 & 28 \\
L3 & 24 & W3 & 2 \\
L4 & 40 & W4 & 30 \\
L5 & 40 & W5 & 2 \\
L6 & 36 & W6 & 1 \\
Lf & 9 & W7 & 6 \\
Ls1 & 4 & Wf & 3.1 \\
Ls2 & 1 & Ws1 & 0.8 \\
Ld & 2 & Ws2 & 2 \\
H & 1.6 & Wd & 2 \\
T & 0.035 & R & 4 \\
\hline
\end{tabular}

\section{CURRENT DISTRIBUTION}

To further visualize the effects of the antenna elements chosen arrangement, as well as those of the used decoupling structure on the electromagnetic coupling amid radiating elements. Fig.8 illustrates the current distribution of the proposed eight-element MIMO antenna at $4 \mathrm{GHz}$ with and without the used decoupling techniques. As depicted in Fig.8 (a) and (b), after the implementation of the decoupling mechanism, the most of current is concentrated on the feedline around the square-slot defected microstrip structure, and an important amount of currents flow through the protruded ground branch decoupling structure which nullifies radiation between the side-by-side spaced antenna elements. Moreover, it is noticeable that current propagation and nearfield coupling between the crossed and perpendicular antenna elements is very weak which proves the efficiency of the chosen isolationenhanced mechanisms.

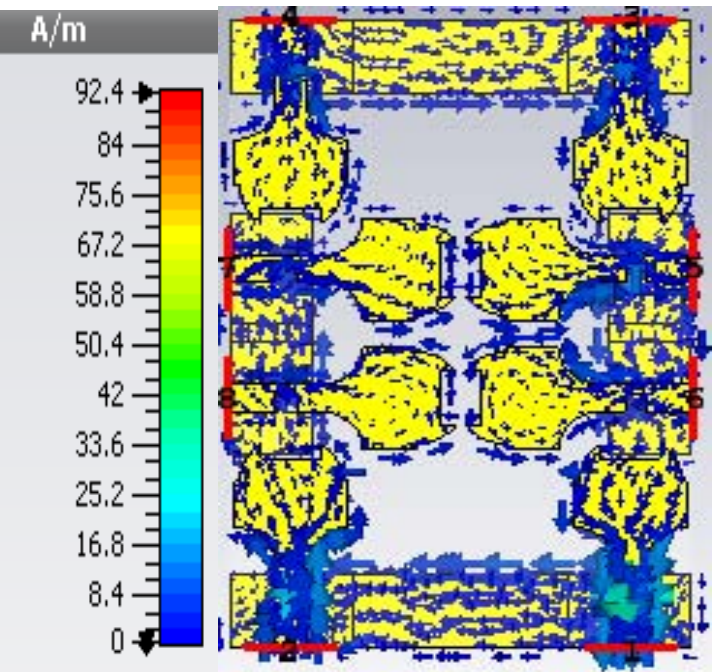

(a) 


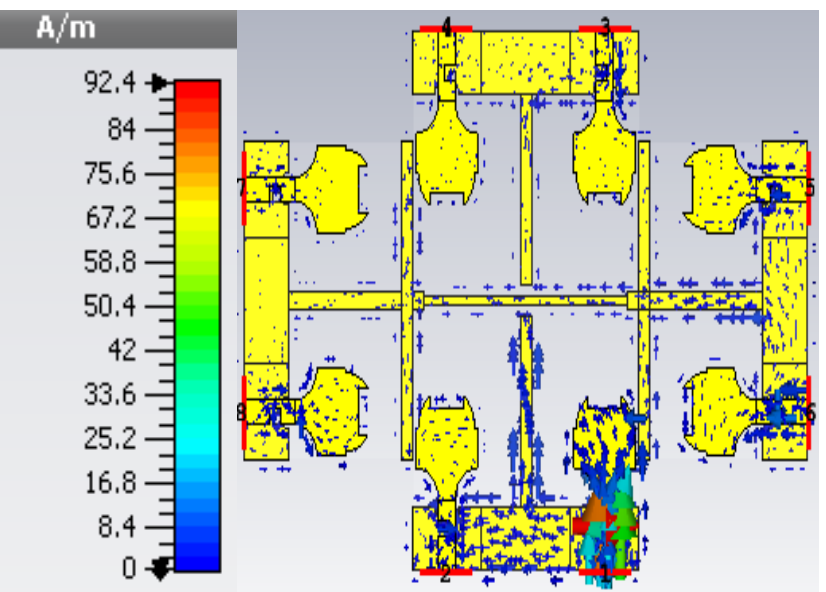

(b)

FIGURE. 8 Current distribution of the proposed MIMO antenna at $4 \mathrm{GHz}$, (a) without the isolation-enhanced technique, (b) with the isolation-enhanced technique

\section{E. EFFICIENCY AND GAIN}

The radiation efficiency is regarded as an important indicator that estimate how lossy the antenna is, since it is known as the ratio of the radiated power to the input power accepted by the antenna [12]. The simulated total efficiency and realized gain of the proposed eight-element MIMO antenna array are plotted in Fig.9. As seen, the presented antenna exhibits a total efficiency that varied between $56 \%$ and $91 \%$ across the UWB operating band. Which means that the studied antenna provides a high efficiency despite of the use of the FR-4 laminate. Moreover, it can retain a good realized gain value which is of about 2 and $6.2 \mathrm{dBi}$ throughout the whole frequency band.

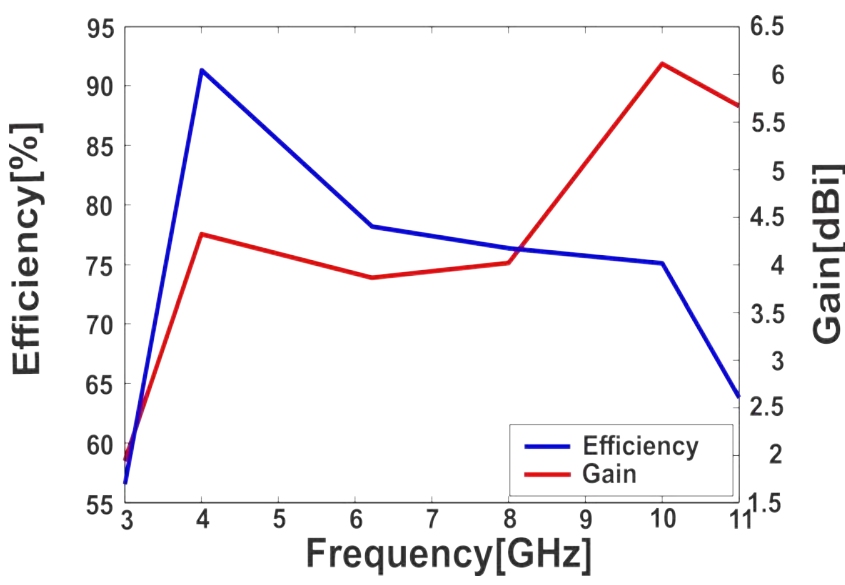

FIGURE. 9 Simulated gain and efficiency of the presented MIMO antenna
In order to validate the simulated results of the studied MIMO antenna system, a prototype of this latter is fabricated using the circuit-board plotter LPKF Protomat. The photographs of the UWB MIMO antenna prototype are depicted in Fig. 10.

Note that the well-known Rohde and Schwarz ZVB 20 network analyzer is used to measure the proposed antenna s-parameters. Fig.11 (a) and (b) show the measured and simulated reflection coefficients and mutual coupling of the presented MIMO antenna array, respectively. As depicted in Fig.11, results indicate that the antenna provide a good impedance matching of about $10 \mathrm{~dB}$ throughout the operating band that range from 2 to $10.6 \mathrm{GHz}$ with high port-to-port isolation which is higher than $15 \mathrm{~dB}$. Note that the slight differences between measured and simulated results due to several factors, include: the used PCB (FR-4) which is more adequate to low frequency application and designs, fabrication imperfection, connectors losses, and soldering. All these factors alter in a negative way on the results.

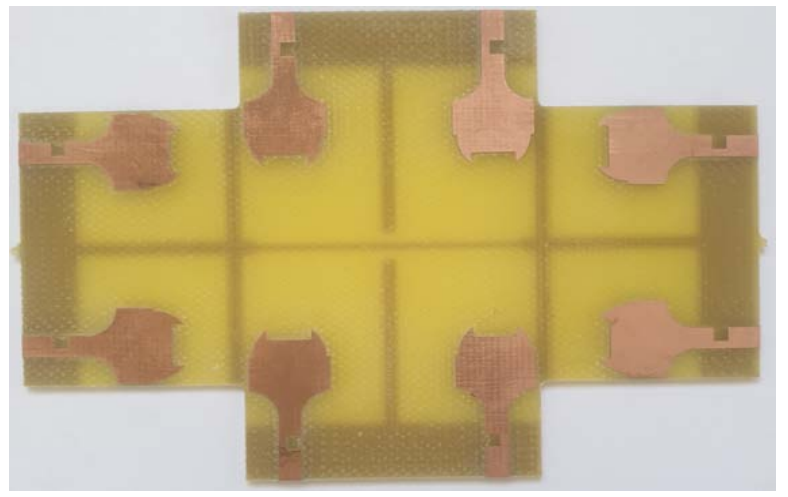

(a)

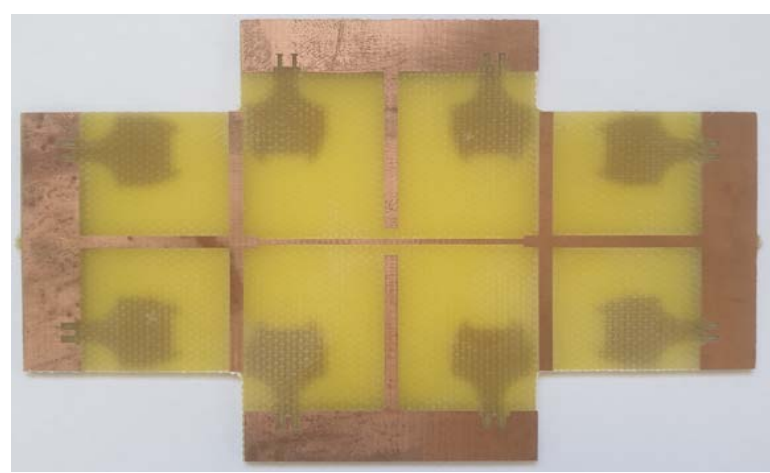

(b)

FIGURE. 10 Photographs of the presented MIMO antenna, (a) top view, (b) bottom view

\section{FABRICATION PROCESS}




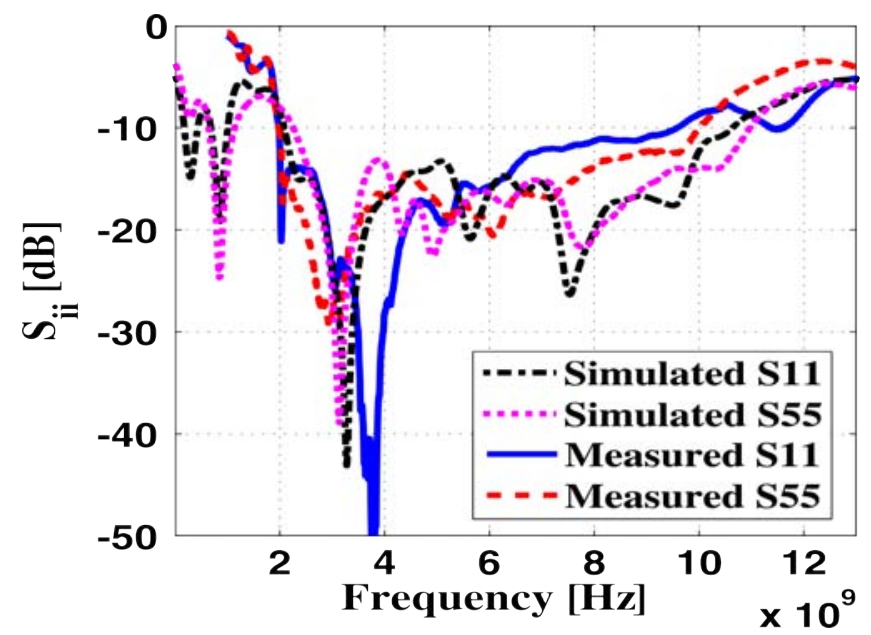

(a)

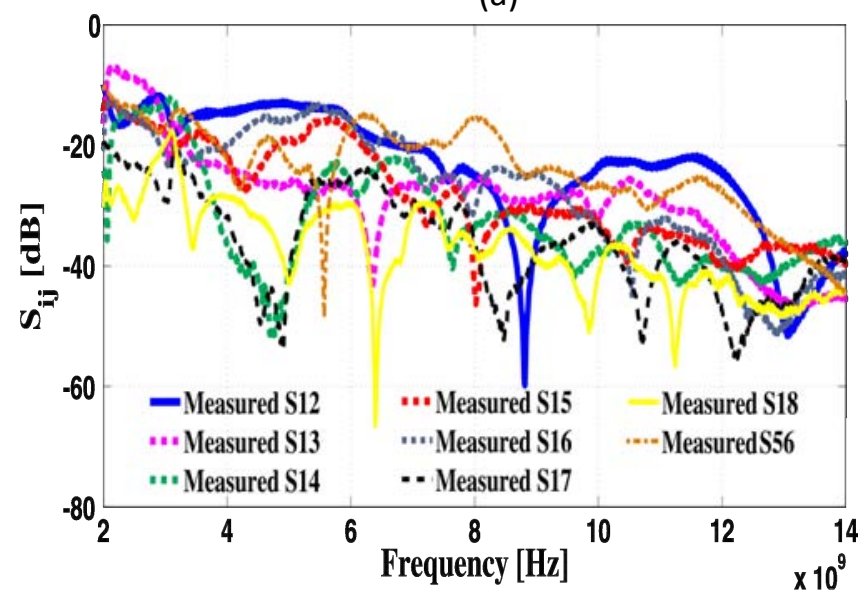

(b)

FIGURE. 11 Simulated and measured reflection coefficients and mutual coupling comparison, (a) simulated $\mathrm{Sij}$, (b) measured $\mathrm{Sij}$

\section{A. ENVELOPE CORRELATION COEFFICIENT}

The ECC is considered as a figure of merit for estimating the diversity capability of a MIMO antenna assembly. It describes how much correlated the antenna elements are in a certain propagation environment by measuring the correlation between the diversity antenna radiation patterns [13]. In brief, it is a quantitative index that reflects the special correlation between two antenna elements received signals.

It is well-known that the ECC value needs to be less than 0.5 which represents the threshold under which the MIMO antenna array can exhibits a good diversity performance. Note that the smaller ECC is, the higher isolation is and vice- versa. In general, the ECC can be calculated using two different methods: s-parameters and far-field pattern based-methods [14].
The envelope correlation coefficient (ECC) based on the complex E-field patterns can be calculated using the following formula [15]:

$$
\rho(i, j)=\frac{\left|\iint_{4 \pi}\left[F_{i}(\theta, \emptyset) * F_{j}(\theta, \emptyset)\right] d \Omega\right|^{2}}{\iint_{4 \pi}\left|F_{i}(\theta, \emptyset)\right|^{2} d \Omega * \iint_{4 \pi}\left|F_{j}(\theta, \emptyset)\right|^{2} d \Omega}
$$

Fig.12 represents the measured envelope correlation coefficients of the studied MIMO antenna system. It can be seen that the measured ECCs are less than 0.15 which is already lower than the threshold (0.5).

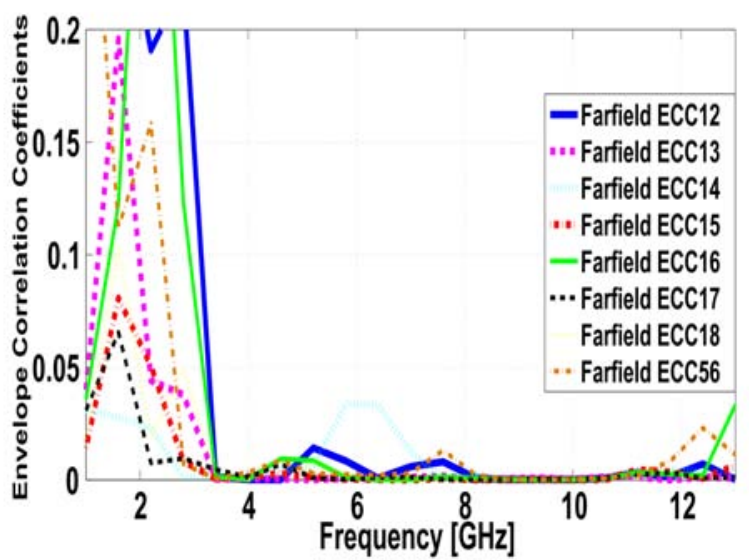

FIGURE. 12 measured envelope correlation coefficients

\section{B. DIVERSITY GAIN}

To furthermore estimate the proposed eight-element MIMO antenna array diversity capability, the diversity gain across the whole UWB operating band is carried out. The diversity gain can be calculated using the envelope correlation coefficient value, as follow [16]:

$$
D G=10 \sqrt{1-\left|\rho_{i, j}\right|^{2}}
$$

Based on this formula, it is obvious that when ECC attains its minimum, the diversity gain attains its maximum. Fig. 13 (a) and (b) depicts the simulated and measured diversity gain of the presented MIMO antenna, respectively. As shown, measured diversity gain which is about 9.4 agree well with simulated (which equal to 10) one except that a slight difference has occurred which is due to the fabrication inaccuracy and measurement conditions. 


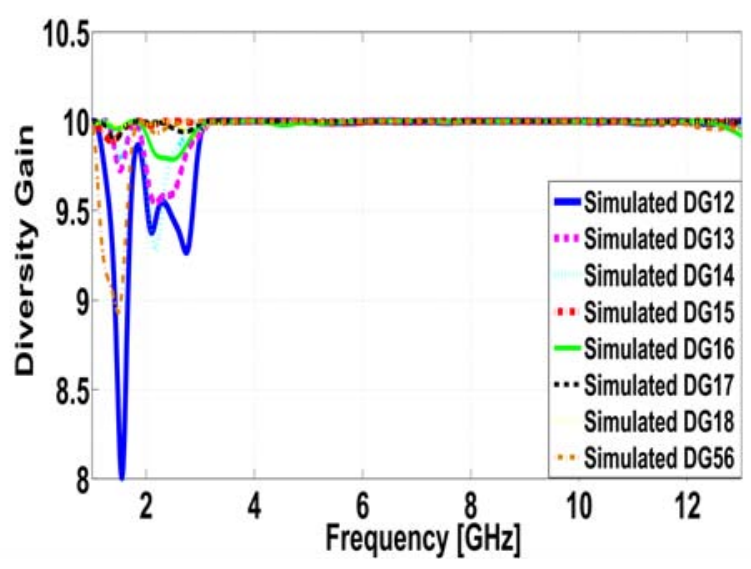

(a)

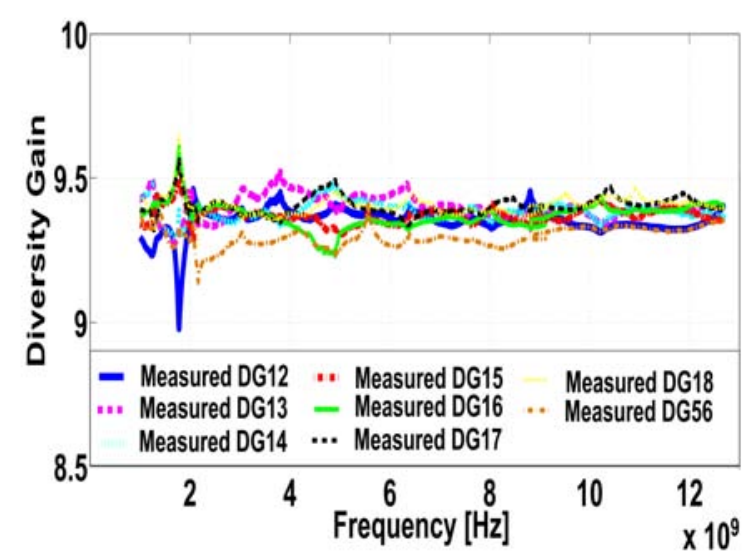

(b)

FIGURE. 13 Simulated and measured diversity gain comparison, (a) simulated DG, (b) measured DG

\section{TOTAL ACTIVE REFLECTION COEFFICIENT}

In Order to predict the overall MIMO system behavior, the Total Active Reflection Coefficient (TARC) that indicate the reflection coefficient of the whole multi-port antenna should be evaluated. For an N-element MIMO antenna assembly, TARC is given by [17]:

$$
\Gamma=\frac{\sqrt{\sum_{i=1}^{N}\left|b_{i}\right|^{2}}}{\sqrt{\sum_{i=1}^{N}\left|a_{i}\right|^{2}}}
$$

Where $a_{i}$ and $b_{i}$ indicate the incident and reflected signals, respectively.

$$
[b]=[S][a]
$$

According to eight-element MIMO antenna system, formula (5) could be written as follow:

$\left[\begin{array}{l}b 1 \\ b 2 \\ b 3 \\ b 4 \\ b 5 \\ b 6 \\ b 7 \\ b 8\end{array}\right]=\left[\begin{array}{llllllll}S 11 & S 12 & S 13 & S 14 & S 15 & S 16 & S 17 & S 18 \\ S 21 & S 22 & S 23 & S 24 & S 25 & S 26 & S 27 & S 28 \\ S 31 & S 32 & S 33 & S 34 & S 35 & S 36 & S 37 & S 38 \\ S 41 & S 42 & S 43 & S 44 & S 45 & S 46 & S 47 & S 48 \\ S 51 & S 52 & S 53 & S 54 & S 55 & S 56 & S 57 & S 58 \\ S 61 & S 62 & S 63 & S 64 & S 65 & S 66 & S 67 & S 68 \\ S 71 & S 72 & S 73 & S 74 & S 75 & S 76 & S 77 & S 78 \\ S 81 & S 82 & S 83 & S 84 & S 85 & S 86 & S 87 & S 88\end{array}\right]\left[\begin{array}{l}a 1 \\ a 2 \\ a 3 \\ a 4 \\ a 5 \\ a b \\ a 7 \\ a \\ a \\ a\end{array}\right]$

Fig.14 illustrates measured and simulated TARC of the proposed MIMO antenna while considering port 1 is the first excited port $(\Theta 1=0)$ and the other ports are excited with following phases: $\Theta 2=40, \Theta 3=60, \Theta 4=80, \Theta 5=90$, $\Theta 6=120, \Theta 7=180, \Theta 8=240$.

It is obvious from figure 15 that the total active reflection coefficient value is less than $-20 \mathrm{~dB}$ across the whole UWB frequency band [3.1-10.6 GHz], which proves more the proposed antenna good performance.

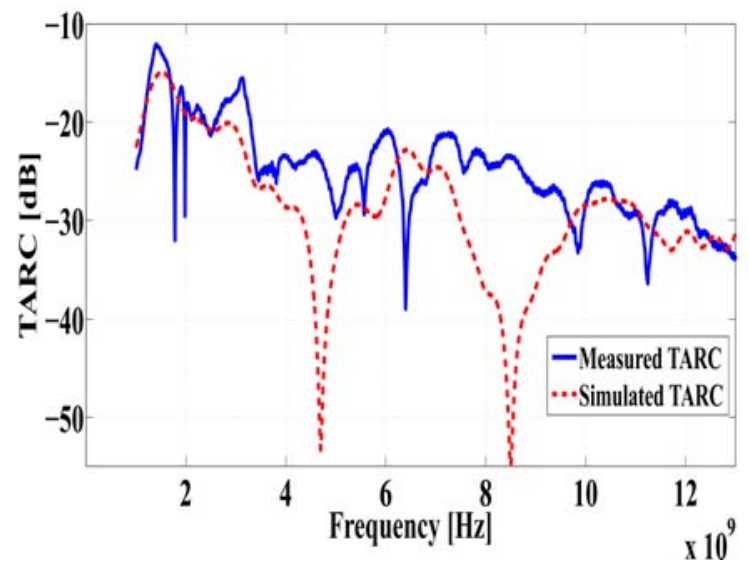

FIGURE. 14 Measured and Simulated Total Active Reflection Coefficient

\section{FARFIELD PATTERN}

Due to symmetry, the radiation patterns for the E- ad Hplanes were investigated when Ant1 is excited and the other ports are terminated with $50 \Omega$ matching loads. Fig.15 represents the simulated and measured radiation characteristics of the presented MIMO antenna at $4 \mathrm{GHz}$, $6.22 \mathrm{GHz}$, and $8 \mathrm{GHz}$. As seen in Fig.15, the radiation patterns get distorted as frequency increases, and they are quasi-omnidirectional to receive signals in an equal way from any direction at both phi $=0^{\circ}$ (H-plane), and phi $=90^{\circ}$ (E-plane) which prevent any temporary loss of signal. Note that, a good agreement is noticed with a slight discrepancy between measured and simulated radiation patterns which is due to the absence of an anechoic chamber. 


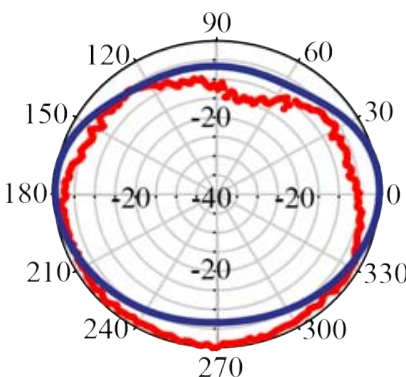

(a) $4 \mathrm{GHz}$

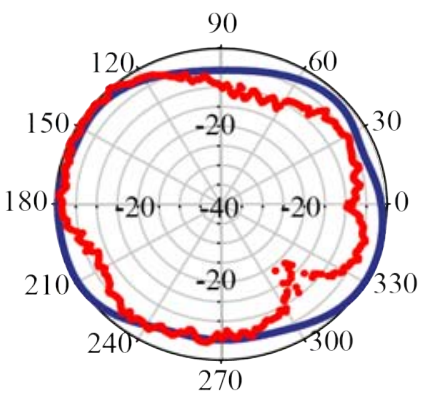

(c) $6.22 \mathrm{GHz}$

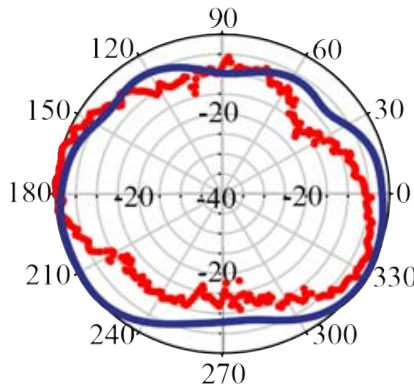

(e) $8 \mathrm{GHz}$

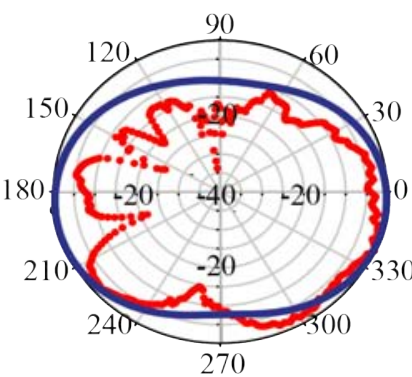

(b) $4 \mathrm{GHz}$

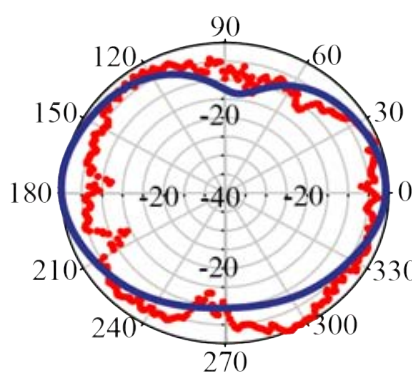

(d) $6.22 \mathrm{GHz}$

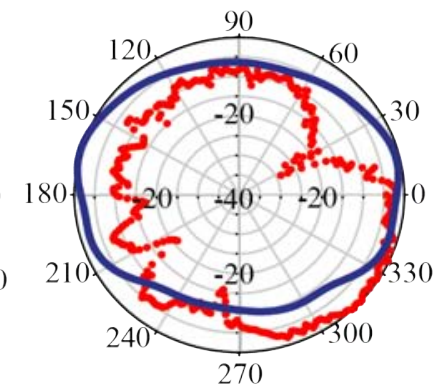

(f) $8 \mathrm{GHz}$

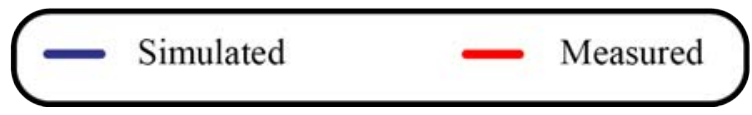

FIGURE. 15 Simulated and measured radiation patterns for $4 \mathrm{GHz}, 6.22$ $\mathrm{GHz}, 8 \mathrm{GHz}$, and $10 \mathrm{GHz}(\mathrm{a}),(\mathrm{c}),(\mathrm{e}) \mathrm{E}-$ plane, and (b), (d), (f) H-plane

\section{PERFORMANCE COMPARISON}

A comparison between the presented MIMO antenna performance and reported ones in terms of number of antenna elements, bandwidth, dimensions, isolation, and efficiency is shown in table III. It can be observed that the proposed MIMO antenna array has an excellent performance and a smaller size compared with reported designs that despite of their low order ( 2 and 4 antenna elements), they are characterized by a high dimension. Therefore, we can say that the presented MIMO antenna system outperforms the reported works.
TABLE III. Performance Comparison

\begin{tabular}{ccccccc}
\hline Ref & No. of Ant & $\begin{array}{l}\text { Bandwidth } \\
(\mathrm{GHz})\end{array}$ & $\begin{array}{c}\text { Dimensions } \\
(\mathrm{mm})\end{array}$ & $\begin{array}{c}\text { Isolation } \\
(\mathrm{dB})\end{array}$ & ECC & $\begin{array}{c}\text { Efficiency } \\
(\%)\end{array}$ \\
\hline $\begin{array}{c}\text { This } \\
\text { work }\end{array}$ & 8 & $2-10.6$ & $100 * 68$ & $>15$ & $<0.15$ & $56-91$ \\
{$[18]$} & 8 & $3-10.6$ & $85 * 85$ & $>15$ & $<0.2$ & - \\
{$[19]$} & 8 & $3.1-12$ & $90 * 90$ & $>10$ & $<0.16$ & - \\
{$[20]$} & 4 & $2.6-10.2$ & $150 * 75$ & $>10$ & $<0.07$ & $60-80$ \\
{$[21]$} & 4 & $2.46-18.2$ & $110 * 119$ & $>20.5$ & $<0.003$ & - \\
{$[22]$} & 2 & $2-10$ & $140 * 140$ & $>25$ & $<0.003$ & $65-80$ \\
{$[23]$} & 2 & $3.1-12$ & $60 * 100$ & $>35$ & - & - \\
\hline
\end{tabular}

\section{CONCLUSION}

This work has successfully reported an eight-element MIMO antenna for UWB applications. The applied diversity techniques prove that in order to cover an ultrawideband operating band using a multi-element MIMO antenna array with a high isolation, spatial and polarization diversity techniques are not sufficient enough. Therefore, another approach has been investigated which is based on the use of a hybrid technique which is simply a combination of a protruded ground branch implementation and a ports-shift technique, and a defected microstrip structure in association with parasitic stubs employment to achieve a high isolation and high impedance matching extended operating frequency band. The simulated and measured results are reasonably matched and satisfy the requirements of an UWB MIMO antenna system, which makes the presented multi-antenna model suitable candidate for UWB applications.

\section{ACKNOWLEDGEMENTS}

This research was partially supported by National School of Applied Sciences, under sciences and advanced technologies laboratory, Abdelmalek Essaadi University, Tetouan, Morocco. 


\section{REFERENCES}

[1] Y. Li, W. Li, and W. Yu, "A multi-band/UWB MIMO/diversity antenna with an enhanced isolation using radial stub loaded resonator," Appl. Comput. Electromagn. Soc. J., vol. 28, no. 1, pp. 8-20, 2013.

[2] S. Zhang, Z. Ying, J. Xiong, and S. He, "Ultrawideband $\mathrm{MIMO}$ /diversity antennas with a tree-like structure to enhance wideband isolation," IEEE Antennas Wirel. Propag. Lett., vol. 8, no. February, pp. 1279-1282, 2009.

[3] J. Jiang, Y. Xia, and Y. Li, "High isolated X-band MIMO array using novel wheel-like metamaterial decoupling structure," Appl. Comput. Electromagn. Soc. J., vol. 34, no. 12, pp. 1829$1836,2019$.

[4] S. Roy, S. Ghosh, and U. Chakarborty, "Compact dual wideband four/eight elements MIMO antenna for WLAN applications," Int. J. RF Microw. Comput Eng., vol. 29, no. 7, pp. 1-15, 2019.

[5] H. Shi, X. Zhang, J. Li, P. Jia, J. Chen, and A. Zhang, "3.6$\mathrm{GHz}$ eight-antenna MIMO array for mobile terminal applications," AEU - Int. J. Electron. Commun., vol. 95, pp. 342-348, 2018.

[6] J. M. Hannula, T. O. Saarinen, A. Lehtovuori, J. Holopainen, and V. Viikari, "Tunable eight element MIMO antenna based on the antenna cluster concept," IET Microwaves, Antennas Propag., vol. 13, no. 7, pp. 959-965, 2019.

[7] M. Y. Li, Z. Q. Xu, Y. L. Ban, Q. L. Yang, and Q. Q. Zhou, "Eight-port dual-polarized MIMO antenna for 5G smartphone applications," APCAP 2016 - 2016 IEEE 5th Asia-Pacific Conf. Antennas Propagation, Conf. Proc., no. February 2018, pp. 195-196, 2017.

[8] M. S. Sharawi, S. K. Podilchak, M. U. Khan, and Y. M. Antar, "Dual-frequency DRA-based MIMO antenna system for wireless access points," IET Microwaves, Antennas Propag., vol. 11, no. 8, pp. 1174-1182, 2017.

[9] J. Guo, L. Cui, C. Li, and B. Sun, "Side-Edge Frame Printed Eight-Port Dual-Band Antenna Array for 5G Smartphone Applications," IEEE Trans. Antennas Propag., vol. 66, no. 12, pp. 7412-7417, 2018.

[10] R. Florencio Díaz, R. Rodríguez Boix, F. Carrasco Yépez, J. Encinar Garcinuño, M. Barba Gea, and G. Pérez Palomino, "Broadband reflectarrays made of cells with three coplanar parallel dipoles," Microw. Opt. Technol. Lett., vol. 56, no. 3, pp. 748-753, 2014.

[11] A. Mchbal, N. Amar Touhami, H. Elftouh, and A. Dkiouak, "Mutual Coupling Reduction Using a Protruded Ground Branch Structure in a Compact UWB Owl-Shaped MIMO Antenna," Int. J. Antennas Propag., vol. 2018, pp. 1-10, 2018.

[12] R. Florencio Díaz, R. Rodríguez Boix, F. Carrasco Yépez, J. Encinar Garcinuño, M. Barba Gea, and G. Pérez Palomino, "Broadband reflectarrays made of cells with three coplanar parallel dipoles," Microw. Opt. Technol. Lett., vol. 56, no. 3, pp. 748-753, 2014.

[13] M. Y. Li, Z. Q. Xu, Y. L. Ban, C. Y. D. Sim, and Z. F. Yu, "Eight-port orthogonally dual-polarised MIMO antennas using loop structures for 5G smartphone," IET Microwaves, Antennas Propag., vol. 11, no. 12, pp. 1810-1816, 2017.

[14] L. Yang, T. Li, and S. Yan, "Highly Compact MIMO Antenna System for LTE/ISM Applications," Int. J. Antennas Propag., vol. 2015, 2015.

[15] M. Y. Li et al., "Eight-Port Orthogonally Dual-Polarized Antenna Array for 5G Smartphone Applications," IEEE Trans. Antennas Propag., vol. 64, no. 9, pp. 3820-3830, 2016.

[16] R. Saleem, M. Bilal, K. B. Bajwa, and M. F. Shafique, "Eightelement UWB-MIMO array with three distinct isolation mechanisms," Electron. Lett., vol. 51, no. 4, pp. 311-313, 2015.

[17] S. K. Palaniswamy, Y. P. Selvam, M. G. N. Alsath, M. Kanagasabai, S. Kingsly, and S. Subbaraj, "3-D Eight-Port Ultrawideband Antenna Array for Diversity Applications,"
IEEE Antennas Wirel. Propag. Lett., vol. 16, no. c, pp. 569572,2017

[18] R. Mathur and S. Dwari, "8-port multibeam planar UWBMIMO antenna with pattern and polarisation diversity", IET Microw., Ant. and Propag., vol. 13, Iss. 13, pp. 2297-2302, 2019.

[19] M. G. N. Alsath et al., "An integrated tri-band/UWB polarization diversity antenna for vehicular networks", IEEE Trans .on Veh.Net., vol. 67, no. 7, pp. 5613-5620, 2018.

[20] N. O. Parchin, H. J. Basherlou, and Y. I. A. Al-yasir, A. M. Abdulkhaleq, and R. A. Abd-Alhameed, "Ultra-Wideband Diversity MIMO Antenna System for Futur Mobile Handsets,' Sensors MDPI, pp. 1-19, 2020.

[21] S. Jayant, and G.Srivastava "Four Elements UWB MIMO Antenna Array," SPIN Int. Conf. pp. 48-54, 2020.

[22] J. A. Tirado-méndez, R. Gómez-villanueva, H. Jardón-Aguilar, E. Fritz-andrade, and A. Rangel-Merino, "UWB MIMO antenna implemented with orthogonal quasi-circular slot dipole radiators," Int. J. RF Microw. Comput. Aided Eng. July, pp. 1$11,2019$.

[23] A. B. Muhajr, J. F. Mahdi, and M. A. Khadhim, "Design of Ultra-Wideband MIMO Antenna for Breast Tumor Detection," IOP Conf. Ser.: Mater. Sci. Eng. 881 012111, 2020. 